\title{
STRONGLY GRADED HEREDITARY ORDERS
}

\author{
JEREMY HAEFNER AND CHRISTOPHER J. PAPPACENA \\ This paper is dedicated to the memory of Professor Dennis Estes.
}

\begin{abstract}
Let $R$ be a Dedekind domain with global quotient field $K$. The purpose of this note is to provide a characterization of when a strongly graded $R$-order with semiprime 1-component is hereditary. This generalizes earlier work by the first author and G. Janusz in (J. Haefner and G. Janusz, Hereditary crossed products, Trans. Amer. Math. Soc. 352 (2000), 3381-3410).
\end{abstract}

Recall that, for a Dedekind domain $R$ with quotient field $K$, an $R$-order in a separable $K$-algebra $A$ is a module-finite $R$-algebra $\Lambda$, contained in $A$, such that $K \Lambda=A$. For a group $G$, we say that the $R$-order $\Lambda$ is strongly $G$-graded provided there is a decomposition $\Lambda=\oplus_{g \in G} \Lambda_{g}$ with $\Lambda_{g} \Lambda_{h}=\Lambda_{g h}$ for all $g, h \in G$. If 1 denotes the identity element of $G$, then $\Lambda_{1}$ is a subring of $\Lambda$, which we denote by $\Delta$. We write $\Lambda=\Delta(G)$ to indicate that $\Lambda$ is strongly $G$-graded with identity component $\Delta$. In this note, we consider the following problem:

The hereditary problem for strongly graded orders: Determine necessary and sufficient conditions on $G$, the grading imposed by $G$, and $\Delta$ to ensure that $\Lambda$ is hereditary.

Our general solution to this problem appears in Theorem 7. The idea of the proof is to use Morita theory to reduce to the case where $\Lambda$ is a crossed product and then apply a result of, 4 , which we describe next.

Recall that a strongly $G$-graded $R$-order $\Lambda=\Delta(G)$ is a crossed product order provided for each $g \in G, \Lambda_{g} \cong \Delta$ as left $\Delta$-modules. In this case, there exist $u_{g} \in \Lambda^{*}$ (the unit group of $\Lambda$ ) such that $\Lambda_{g}=\Delta u_{g}$ for all $g \in G$. Moreover, there exist a group homomorphism $\alpha: G \rightarrow \operatorname{Aut}_{R}(\Delta)$ (the "action of $G$ on $\Delta$ ") and a cocycle $\tau \in Z^{2}\left(G, R^{*}\right)$ (the "twisting of the action of $G$ ") such that the multiplication in $\Lambda$ is given by $u_{g} \delta=\alpha(g)(\delta) u_{g}$ for $\delta \in \Delta$, and $u_{g} u_{h}=\tau(g, h) u_{g h}$. (See [6] for more details on this construction.)

If $\Delta(G)$ is a crossed product order with action $\alpha$, we say that a subgroup $H$ of $G$ acts as central outer automorphims of $\Delta$ provided $\alpha(H) \cap \operatorname{Inn}(\Delta)=1$. The main result of 4 is that, if $\Lambda=\Delta(G)$ is a crossed product order, then $\Lambda$ is hereditary if and only if $\Delta$ is hereditary and, for each maximal ideal $\mathfrak{m}$ of $R$ containing a prime divisor $p$ of $|G|$, any $p$-Sylow subgroup of $G$ acts as central outer automorphisms of $\hat{\Delta}_{\mathfrak{m}}$.

Definition 1. Given a strongly $G$-graded ring $\Lambda=\Delta(G)$ and $g \in G$, we say $g$ is inner on $\Delta$ provided $\Lambda_{g} \cong \Delta$ as $\Delta$-bimodules. Otherwise, $g$ is outer on $\Delta$. For a

Date: November 3, 2018.

1991 Mathematics Subject Classification. 16G30, 16H05, 16S35, 16W20, 16W50.

Key words and phrases. order, hereditary, strongly graded, inner and outer grading.

This research was funded in part by a Baylor University Research Grant. 
subgroup $H$ of $G$, set

$$
\operatorname{Inn}_{\Delta}(H)=\{h \in H: h \text { is inner on } \Delta\}
$$

We say that $H$ is inner on $\Delta$ or inner grades $\Delta$ if $\operatorname{Inn}_{\Delta}(H)=H$ and it is outer on $\Delta$ or outer grades $\Delta$ provided $\operatorname{Inn}_{\Delta}(H)=1$.

We remark that the above definitions do indeed generalize the classical notion of inner actions. To see this, suppose $\Lambda=\Delta(G)$ is a crossed product order with group action $\alpha: G \rightarrow \operatorname{Aut}_{R}(\Delta)$ such that $\alpha(g) \in \operatorname{Inn}_{R}(\Delta)$ for some $g \in G$. Then it is easy to see that $\Lambda_{g}=\Delta u_{g} \cong \Delta$ as bimodules. Hence, if $g$ acts as an inner automorphism on $\Delta$ in the classical sense, it is inner on $\Delta$ in the sense defined above.

Lemma 2. Let $\Lambda=\Delta(G)$ be strongly graded by $G$. Then, for any subgroup $H$ of $G, \operatorname{Inn}_{\Delta}\left(g^{-1} H g\right)=g^{-1} \operatorname{Inn}_{\Delta}(H) g$.

Proof. Suppose that $h \in \operatorname{Inn}_{\Delta}(H)$. Then $\Delta_{h} \cong \Delta_{1}$ as bimodules. It follows that $\Delta_{g^{-1}} \Delta_{h} \Delta_{g} \cong \Delta_{1}$ as bimodules as well. This shows that $g^{-1} \operatorname{Inn}_{\Delta}(H) g \subseteq$ $\operatorname{Inn}_{\Delta}\left(g^{-1} H g\right)$. For the converse, let $\Delta_{g^{-1} h g} \cong \Delta_{1}$ as bimodules. Since $\Delta$ is strongly graded, $\Delta_{g^{-1} h g}=\Delta_{g^{-1}} \Delta_{h} \Delta_{g}$. It follows that $\Delta_{h} \cong \Delta_{1}$ as bimodules, proving the reverse inclusion.

Lemma 3. Suppose that $R$ is a complete DVR, and that $\Lambda=\Delta(G)$ is a stronglygraded $R$-order such that $\Delta$ is prime and basic. Then $\Delta(G)$ is a crossed product order.

Proof. Since $R$ is a complete DVR, $\Delta$ is semiperfect. Consequently there exist only finitely many indecomposable projective left $\Delta$-modules up to isomorphism. Let $\left\{P_{1}, \ldots, P_{t}\right\}$ be representatives for these isomorphism classes. Then there exist positive integers $m_{i}$ such that $\Delta \cong \oplus_{i=1}^{t} P_{i}^{\left(m_{i}\right)}$ as left $\Delta$-modules.

For $g \in G$, we have that $\Lambda_{g} \in \operatorname{Pic}(\Delta)$, because $\Lambda$ is strongly graded. This implies that $\Lambda_{g}$, viewed as a left $\Delta$-module, is isomorphic to a direct sum of the $P_{i}$. So, we may write $\Lambda_{g} \cong \oplus_{i=1}^{t} P_{i}^{\left(n_{i}\right)}$, where the $n_{i}$ are a priori nonnegative integers. Now, since $\Lambda_{g}$ is a progenerator and $\operatorname{End}\left(\Lambda_{g}\right) \cong \Delta$ as rings, a combinatorial argument shows that in fact $m_{i}=n_{i}$ for all $i$, so that $\Lambda_{g} \cong \Delta$ as left $\Delta$-modules. Thus $\Lambda$ is a crossed product.

We can now state and prove the prime case of our solution to the hereditary problem. We use the following notation for the remainder of the paper. $R$ denotes a Dedekind domain whose quotient field $K$ is a global field and $A$ denotes a separable $K$-algebra. For a maximal ideal $\mathfrak{m}$ of $R, \hat{R}_{\mathfrak{m}}$ denotes the completion of $R$ at $\mathfrak{m}$. Similarly, $\Delta$ denotes an $R$-order in $A$, and $\hat{\Delta}_{\mathfrak{m}}$ denotes the $\hat{R}_{\mathfrak{m}}$-order $\hat{R}_{\mathfrak{m}} \otimes_{R} \Delta$.

Theorem 4. Assume that $A$ is simple (so $\Delta$ is prime). Then $\Lambda$ is hereditary if and only if $\Delta$ is hereditary and, for each maximal ideal $\mathfrak{m}$ of $R$ containing a prime divisor $p$ of $|G|$, some (hence every) p-Sylow subgroup of $G$ is outer on $\hat{\Delta}_{\mathfrak{m}}$.

Proof. First note that the induction functor $\Lambda \otimes_{\Delta}-: \Delta-\bmod \rightarrow \Lambda-\bmod$ is separable [4, Proposition 2.2]. It follows by [ 4 . Proposition 2.3] that if $\Lambda$ is hereditary, then so is $\Delta$. Thus, for the remainder of the proof, we assume that $\Delta$ is hereditary. Next observe that any two $p$-Sylow subgroups of $G$ are conjugate. Thus, by Lemma 2, it suffices to verify that $\operatorname{Inn}_{\hat{\Delta}_{\mathfrak{m}}}(P)=1$ for a single $p$-Sylow subgroup $P$. By $\llbracket$. 
Theorem 40.5], $\Lambda$ is hereditary if and only if $\hat{\Lambda}_{\mathfrak{m}}$ is hereditary for each maximal ideal $\mathfrak{m}$. We show that $\Lambda$ is hereditary if and only if $\hat{\Lambda}_{\mathfrak{m}}$ is hereditary for those maximal ideals $\mathfrak{m}$ containing a prime divisor of $|G|$.

To see this, assume $\hat{\Lambda}_{\mathfrak{m}}$ is hereditary for those maximal ideals $\mathfrak{m}$ containing a prime divisor of $|G|$. Fix an arbitrary maximal ideal $\mathfrak{m}$ of $R$. If it contains no prime

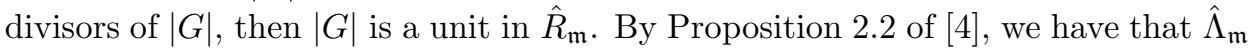
is a separable extension of $\hat{\Delta}_{\mathfrak{m}}$. Since $\Delta$ is hereditary, so is $\hat{\Delta}_{\mathfrak{m}}$ and it follows from [4. Proposition 2.3] that $\hat{\Lambda}_{\mathfrak{m}}$ is hereditary. Thus, to verify whether $\Lambda$ is hereditary, it suffices to check $\hat{\Lambda}_{\mathfrak{m}}$ at those maximal ideals $\mathfrak{m}$ containing prime divisors of $|G|$.

Assume that $\mathfrak{m}$ contains a prime divisor $p$ of $|G|$, and fix a $p$-Sylow subgroup $P$ of $G$. For ease of notation, we write $\hat{R}$ for $\hat{R}_{\mathfrak{m}}$, etc. Since $\hat{\Delta}$ is prime hereditary, there is an idempotent $e$ of $\hat{\Delta}$ such that $e \hat{\Delta} e$ is basic hereditary. We claim that $e \hat{\Lambda} e$ is a strongly $G$-graded order with components $e \hat{\Lambda}_{x} e$ for $x \in G$. To see this, fix homogeneous components $e \hat{\Lambda}_{g} e, e \hat{\Lambda}_{h} e$. Then,

$$
\begin{aligned}
e \hat{\Lambda}_{g} e \cdot e \hat{\Lambda}_{h} e & =e \hat{\Lambda}_{g} e \hat{\Lambda}_{h} e \\
& =e \hat{\Lambda}_{g} \hat{\Lambda}_{1} e \hat{\Lambda}_{1} \hat{\Lambda}_{h} e \quad(\text { since } \hat{\Lambda} \text { is strongly } G \text {-graded) } \\
& =e \hat{\Lambda}_{g} \hat{\Delta} \hat{\Lambda}_{h} e \quad\left(\text { since } \hat{\Lambda}_{1}=\hat{\Delta} \text { and } \hat{\Delta} e \hat{\Delta}=\hat{\Delta}\right) \\
& =e \hat{\Lambda}_{g h} e \quad \text { (since } \hat{\Lambda} \text { is strongly } G \text {-graded) }
\end{aligned}
$$

which shows that $e \hat{\Lambda} e$ is strongly graded.

The orders $\hat{\Lambda}$ and $e \hat{\Lambda} e$ are Morita equivalent via the pair of graded progenerators $\hat{\Lambda} e$ and $e \hat{\Lambda}$. In addition, this equivalence preserves, in a certain sense, the grading of the two orders. This is an example of what is called a graded equivalence; see [2] for more information on graded equivalences.

By Morita equivalence, $\hat{\Lambda}$ is hereditary if and only if $e \hat{\Lambda} e$ is hereditary. Now, the identity component of $e \hat{\Lambda} e$ is $e \hat{\Delta} e$, which is basic, prime and hereditary. Since $e \hat{\Lambda} e$ is strongly $G$-graded, we see by Lemma 3 that $e \hat{\Lambda} e$ is a crossed product order. Hence, we may apply [4, Theorem 6.8] to conclude that $e \hat{\Lambda} e$ is hereditary if and only if $P$ acts as central outer automorphisms. As we have remarked above, this is equivalent to saying that $P$ outer grades $e \hat{\Lambda} e$. To finish the proof, we note that $P$ outer grades $e \hat{\Lambda} e$ if and only if $P$ outer grades $\hat{\Lambda}$, because $\hat{\Lambda}_{x} \cong \hat{\Delta}$ as bimodules if and only if $e \hat{\Lambda}_{x} e \cong e \hat{\Delta} e$ for any $x \in P$. (This uses the fact that $\hat{\Lambda} e$ and $e \hat{\Lambda}$ induce a graded equivalence between $\hat{\Lambda}$ and $e \hat{\Lambda} e$.) Thus, $\hat{\Lambda}$ is hereditary if and only if $\operatorname{Inn}_{\hat{\Lambda}}(P)=1$.

The above proof requires us to reduce to the case when $\Delta$ is basic so that $\Lambda$ is a crossed product order. We present an example that shows that, even over a complete DVR, a strongly graded order with non-basic hereditary 1-component need not be a crossed product order. Thus Lemma 3 is the best possible, and the proof of Theorem 1 cannot be simplified in this regard. The example depends on the following basic construction technique for strongly graded rings, which is a special case of [5, p. 23].

Construction. Let $\Delta$ be a ring, and let $X \in \operatorname{Pic}(\Delta)$ have finite order $n$. Let $G$ denote the cyclic group of order $n$ with generator $g$. We construct a $G$-strongly graded $\operatorname{ring} \Lambda$ with 1-component $\Delta$ as follows: Set $\Lambda=\oplus_{i=1}^{n} \Lambda_{g^{i}}$, where $\Lambda_{g^{i}}=X^{i}$, and define the multiplication by the tensor product. In other words, fix isomorphisms 
$X^{i} \otimes X^{j} \cong X^{i+j}$ which are compatible in the obvious sense. Then, given homogeneous elements $x_{i}, x_{j}$ in $\Lambda_{g^{i}}, \Lambda_{g^{j}}$, respectively, we define $x_{i} \cdot x_{j}=x_{i} \otimes x_{j} \in X^{i+j}$ (using the fixed isomorphism). Note that $\Lambda_{1}=\Lambda_{g^{0}}=X^{0} \cong \Delta$, so the 1-component is $\Delta$, as claimed. Note also the grading is strong, as $X^{i} \otimes X^{j} \cong X^{i+j}$ for all $i, j$ by construction.

Example 5 (Strongly graded orders need not be crossed products). Let $\Delta$ be a nonbasic hereditary order over a complete DVR, and let $\Gamma$ be the associated basic order $e \Delta e$. Now, $\operatorname{Picent}(\Delta) \cong \operatorname{Picent}(\Gamma)$ is cyclic (say of order $n$ ) generated by $\operatorname{rad}(\Delta)$ $(\operatorname{respectively} \operatorname{rad}(\Gamma))$. The fact that it is cyclic is in [4], and the fact that it is generated by the radical follows from [7], where it is shown that $\operatorname{rad}(\Delta)$ has the correct order. Now, if $\Delta$ is not basic, then $\operatorname{rad}(\Delta)^{k}$ is not principal as a left ideal for any $1 \leq k<n$, and $\operatorname{so} \operatorname{rad}(\Delta)^{k} \neq \Delta$ as bimodules for any $1 \leq k<n$. Thus, the $\mathbb{Z} / n \mathbb{Z}$-strongly graded order $\Delta(G)=\oplus_{k=0}^{n-1} \operatorname{rad}(\Delta)^{k}$ is not a crossed product order, even though $e \Delta(G) e$ is.

Having dealt with the prime case, we turn our attention to the semiprime case. To begin, we investigate the action of $G$ that is imposed on the central idempotents of $\Delta$. We fix the following notation.

Notation. Given $\Lambda=\Delta(G)$, suppose that $\Delta=\Delta_{1} \oplus \cdots \oplus \Delta_{t}$ is a direct sum of prime rings. Let $e_{1}, \ldots, e_{t}$ denote the orthogonal central idempotents of $\Delta$. Then the group $G$ acts on the $e_{i}$, by $e_{i} \Lambda_{g}=\Lambda_{g} e_{g(i)}$. (This is a special case of the action of $\operatorname{Pic}(\Delta)$ on the center of $\Delta$; see [1, §55].) Suppose that this action partitions $\left\{e_{i}\right\}$ into $m$ orbits, and let $\varepsilon_{1}, \ldots, \varepsilon_{m}$ be a set of representatives for the equivalence classes under this action. Finally, let $G_{i}$ denote the stabilizer of $\varepsilon_{i}$.

Lemma 6. Assume the above notation.

(a) $\Lambda$ is Morita equivalent to $\oplus_{i=1}^{m} \varepsilon_{i} \Lambda \varepsilon_{i}$.

(b) Each $\varepsilon_{i} \Lambda \varepsilon_{i}$ is strongly graded by $G_{i}$, with identity component $\varepsilon_{i} \Delta \varepsilon_{i}$, a prime ring.

Proof. This is proven in [3, Theorem 5.4], under the assumption that $\Delta$ is maximal. However, examining the proof, we see that the above is true without this hypothesis.

Theorem 7. Let $R$ be a Dedekind domain with global quotient field $K$. Let $A$ be a semisimple $K$-algebra, and $\Delta$ be an $R$-order in $A$. (Note that $\Delta$ is necessarily semiprime.) Suppose that $\Lambda=\Delta(G)$ is a strongly graded $R$-order. Then $\Delta(G)$ is hereditary if and only if $\Delta$ is hereditary, and, in the notation of Lemma 6 , the following conditions hold, for $1 \leq i \leq m$.

For each maximal ideal $\mathfrak{m}$ of $R$ containing a prime divisor $p$ of $\left|G_{i}\right|, \operatorname{Inn}_{\varepsilon_{i} \hat{\Delta}_{\varepsilon_{i}}}(P)=1$ for some (hence every) $p$-Sylow subgroup $P$ of $G_{i}$.

Proof. Note that, as in the proof of Theorem 1 , we conclude that $\Delta$ being hereditary is a necessary condition. Thus, we may assume $\Delta$ is hereditary, and then $\Delta$ decomposes as a direct sum of prime rings. Hence, Lemma 6 applies.

Since $\Lambda$ is Morita equivalent to $\oplus_{i=1}^{m} \varepsilon_{i} \Lambda \varepsilon_{i}$, it follows that $\Lambda$ is hereditary if and only if each $\varepsilon_{i} \Lambda \varepsilon_{i}$ is. Now, each $\varepsilon_{i} \Lambda \varepsilon_{i}$ has prime identity component, so that Theorem 目applies. 
We close this paper with some remarks and examples. First, the decomposition $\oplus_{i} \varepsilon_{i} \Lambda \varepsilon_{i}$ depends upon the choice of the representatives $\varepsilon_{i}$ of the orbits of $\left\{e_{1}, \ldots, e_{t}\right\}$. If we chose different representatives $\varepsilon_{i}^{\prime}$, then the new stabilizer groups $G_{i}^{\prime}$ would be conjugate to the original groups $G_{i}$. If we choose $p$-Sylow subgroups $P, P^{\prime}$ of $G_{i}, G_{i}^{\prime}$ respectively, then $\operatorname{Inn}_{\varepsilon_{i} \hat{\Delta} \varepsilon_{i}}(P)=1$ if and only if $\operatorname{Inn}_{\varepsilon_{i}^{\prime} \hat{\Delta} \varepsilon_{i}^{\prime}}\left(P^{\prime}\right)=1$, by Lemma 2. Thus, the choice of representatives does not affect the application of Theorem 7 .

Second, the statement of Theorem 7 requires checking whether or not $\operatorname{Inn}_{\hat{\Delta}}(P)=$ 1 at various completions. It is not enough to assume that $\operatorname{Inn}_{\Delta}(P)=1$, i.e. that $P$ outer grades $\Delta$ globally, because the property of being outer is not a local-global property. The next example illustrates this fact.

Example 8 (Outer grading is not a local-global property). Let $R$ be the ring of Gaussian integers $\mathbb{Z}[i]$, and let $K$ denote the quotient field $\mathbb{Q}(i)$. The prime integer 5 is contained in exactly two ideals of $R: \mathfrak{p}=(1+2 i)$ and $\mathfrak{q}=(1-2 i)$. Let $I$ denote the ideal (5), and let $\Delta$ denote the tiled order

$$
\Delta=\left(\begin{array}{ccccc}
R & R & R & R & R \\
I & R & R & R & R \\
I & I & R & R & R \\
I & I & I & R & R \\
I & I & I & I & R
\end{array}\right)
$$

We first compute Picent $(\Delta)$. By Fröhlich's Theorem [1] Theorem 55.25], there is an isomorphism

$$
\operatorname{Picent}(\Delta) \cong \bigoplus_{\mathfrak{m} \text { maximal }} \operatorname{Picent}\left(\hat{\Delta}_{\mathfrak{m}}\right)
$$

(Here we are using that $R$ is a PID.) Note that, if $\mathfrak{m} \neq \mathfrak{p}, \mathfrak{q}$, then $\hat{I}_{\mathfrak{m}} \cong \hat{R}_{\mathfrak{m}}$, and so $\hat{\Delta}_{\mathfrak{m}}=M_{5}\left(\hat{R}_{\mathfrak{m}}\right)$. In particular, Picent $\left(\hat{\Delta}_{\mathfrak{m}}\right)=0$ if $\mathfrak{m} \neq \mathfrak{p}, \mathfrak{q}$.

If $\mathfrak{m}=\mathfrak{p}$, then $\hat{\Delta}_{\mathfrak{p}}$ is the unique basic hereditary order in $M_{5}(\hat{K})$, and so $\operatorname{Picent}\left(\hat{\Delta}_{\mathfrak{p}}\right) \cong \mathbb{Z} / 5 \mathbb{Z}$, by [7] Theorem 39.18]. Similarly, Picent $\left(\hat{\Delta}_{\mathfrak{q}}\right) \cong \mathbb{Z} / 5 \mathbb{Z}$. Thus, $\operatorname{Picent}(\Delta) \cong \mathbb{Z} / 5 \mathbb{Z} \oplus \mathbb{Z} / 5 \mathbb{Z}$.

Now, let $X$ be the bimodule generating the subgroup $0 \oplus \mathbb{Z} / 5 \mathbb{Z}$ of $\operatorname{Picent}(\Delta)$ (i.e. the component corresponding to $\left.\hat{\Delta}_{\mathfrak{q}}\right)$, and form the strongly $\mathbb{Z} / 5 \mathbb{Z}$-graded order $\Lambda$, where $\Lambda_{g^{i}}=X^{i}$. Note that, globally, $\Lambda$ is outer graded (because $X^{i} \cong \Delta$ as bimodules if and only if $i=0$ ). However, if we pass to the completion at $\mathfrak{p}$, then $\hat{X}_{\mathfrak{p}} \cong \hat{\Delta}_{\mathfrak{p}}$ as bimodules, by construction. Thus, it is possible for a global outer grading to become inner at the completion.

Finally, Theorem 7 requires checking the condition on the grading at each $\varepsilon_{i} \Lambda \varepsilon_{i}$ (in the notation of Lemma 6), rather than simply checking the grading on $\Lambda$. That is, it is not enough to verify that $\operatorname{Inn}_{\hat{\Lambda}}(P)=1$ for $p$-Sylow subgroups of each $G_{i}$ (or of $G$ ). This is because the Morita equivalence between $\Lambda$ and $\oplus_{i=1}^{m} \varepsilon_{i} \Lambda \varepsilon_{i}$ is not a graded equivalence. Hence, the property of being an outer grading is not preserved under this correspondence. Our last example illustrates this.

Example 9 (Passing to $\varepsilon_{i} \Lambda \varepsilon_{i}$ can change the grading). Let $\Delta$ be a prime, hereditary order, and let $\Delta^{(d)}$ denote the direct sum of $d$ copies of $\Delta$. The symmetric group $S_{d}$ acts on $\Delta^{(d)}$ by permuting coordinates, so that we may form the crossed product (with trivial twisting) $\Delta^{(d)}\left(S_{d}\right)$ relative to this action. In the notation of 
Lemma 6, $G=S_{d}$ acts transitively on $\left\{e_{1}, \ldots, e_{d}\right\}$. Let us fix $\varepsilon_{1}=e_{1}$, so that $G_{1}=S_{d-1}$, embedded in $S_{d}$ as those permutations that fix the first coordinate.

It is straightforward to compute that $e_{1} \Delta^{(d)}\left(S_{d}\right) e_{1} \cong \Delta S_{d-1}$, the ordinary group ring of $S_{d-1}$ over $\Delta$. Note that, for any $p$-Sylow subgroup $P$ of $S_{d-1}, \operatorname{Inn}_{\Delta S_{d-1}}(P)=$ $P$. However, if we view $P$ as a subgroup of $S_{d}$, then $\operatorname{Inn}_{\Delta^{(d)}\left(S_{d}\right)}(P)=1$. This is because, for $\pi \in P, \Delta^{(d)}\left(S_{d}\right)_{\pi} \not \Delta^{(d)}$ as bimodules. (The action on the right is twisted by $\pi$, and $\pi$ is not an inner automorphism of $\Delta$.) Thus, it is necessary when applying Theorem 7 to consider the grading on each $\varepsilon_{i} \Lambda \varepsilon_{i}$, and not simply the grading on $\Lambda$.

\section{REFERENCES}

[1] C. Curtis and I. Reiner, Methods of Representation Theory with Applications to Finite Groups and Orders, Volume II, Wiley and Sons, New York, 1987.

[2] J. Haefner, Graded equivalence with applications, J. Algebra 172 (1995), 385-424.

[3] J. Haefner, Reduction techniques for strongly graded rings of finite representation type I: Bounded functors and graded equivalence, J. Algebra 194 (1997), 567-593.

[4] J. Haefner and G. Janusz, Hereditary crossed products, Trans. Amer. Math. Soc. 352 (2000), 3381-3410.

[5] C. Năstăsescu and F. Van Oystaeyen, Graded Ring Theory, North Holland, Amsterdam, 1982.

[6] D. Passman, Infinite Crossed Products, Academic Press, New York, 1989.

[7] I. Reiner, Maximal Orders, Academic Press, New York, 1975.

Department of Mathematics, University of Colorado, Colorado Springs, CO 80933 7150

E-mail address: haefner@math.uccs.edu

Department of Mathematics, Baylor University, Waco, TX 76798

E-mail address: Chris_ Pappacena@baylor.edu 